\title{
Untersuchungen über die Eiweisskörper der Milch.
} Von

\author{
F. Wilh. Zahn.
}

Aus dem physiologischen Institut von Herrn Geh. Rath Prof. Helmholtz in Heidelberg.

Die Milch, als Secret der weiblichen Brustdrüse, ist seit geraumer Zeit Objekt mannigfacher Untersuchungen gewesen. Trotz vieler Resultate, die sich in Folge dessen darüber ergeben haben, kann nichtsdestoweniger dieses Feld keineswegs als abgeschlossen betrachtet werden, denn über die in der Milchdrüse vor sich gehenden physiologischen Vorgänge existiren bis jetzt kaum Vermuthungen. Aus diesem Grunde und weil dieses Secret für den Säugling von so ungemeiner Wichtigkeit ist, dürften neue und wiederholte Untersuchungen über diesen Gegenstand vielleicht nicht unwillkommen sein. Ganz besonders gilt dies für die Eiweisskörper der Milch, welche als die höchstorganisirten Produkte der progressiven Stoffmetamorphose so sehr leicht Umwandlungen erleiden, wodurch sie der physiologischen Untersuchung so äusserst schwer zugänglich werden. Der Erforschung ihrer Eigenschaften waren nachfolgende Versuche hauptsächlich bestimmt, und beruhen diese vornehmlich auf dem Principe mechanischer Trennung dieser Körper von einander.

Bis jetzt nimmt man im Allgemeinen an, dass zwei verschiedene Eiweisskörper in der Milch vorkommen, das Albumin und das Caseïn. Sie waren auch Gegenstand dieser Untersuchungen gewesen, da die Existenz eines dritten, von Millon und Commaille ${ }^{1}$ ) angegebenen Eiweisskörpers, das Lactoproteïn, erst von dieser Seite in der Milch nachgewiesen wurde, aus verschiedenen Gründen aber in den Bereich dieser Untersuchungen nicht hereingezogen werden konnte.

\section{Das Albumin der Milch.}

Die Proteïnkörper der Milch stammen zweifelsohne von dem Serumalbumin des Blutes. Es ist auch die im Colostrum der Säugethiere vorkommende Eiweisssubstanz vom Serumalbumin in Nichts verschieden. In der Milch des Schweines findet sich, wie Joly und

1) Analyse du lait. Comptes rendus. 1864. II. pag. 396. 
Filhol ${ }^{1}$ ) nachgewiesen haben, nur der in der Hitze coagulirende Eiweisskörper, während in der Milch anderer Thiere neben diesem noch ein anderer beim Erwärmen nicht gerinnender nicht nur angetroffen wird, sondern durch seine Menge dermassen vorwiegt, dass das Vorhandensein des ersteren nur schwer nachzuweisen ist und dadurch selbst zweifelhaft werden kann. Dieser Umstand ist auch die Ursache, dass über das Vorkommen dieses Stoffes in normaler Milch so entgegengesetzte Meinungen geltend gemacht werden konnten. Da bei der frischen normalen Milch, mit wenigen Ausnahmen, Gerinnungserscheinungen beim Erwärmen derselben nicht wahrgenommen werden konnten, und man besagten gerinnungsfähigen Körper erst nach Ausfällung des in der Hitze nicht gerinnenden, des Caseïn, und der Fette erhielt, so konnte man mit demselben Recht annehmen, dass er ein Produkt des Fällungsmittels sei, als dass er schon vorher bestanden habe. Ein hauptsächliches Hinderniss zur endgültigen Entscheidung dieser Frage, war die Unfiltrirbarkeit der Milch. Erst Hop $\mathrm{pe}^{2}$ ) gelang es mittelst Filtration frischer Milch durch einen, vorher mit Wasser und Alkohol gereinigten menschlichen Ureter ein Filtrat von geringer Opalescenz herzustellen, aus welchem durch Erwärmen bei $70-75^{\circ}$ ein gerinnbarer Eiweisskörper abgeschieden werden konnte, und hält er damit den "Beweis der Anwesenheit des Albumin in der frischen normalen Milch « für gegeben. Obwohl diese Filtrirmethode vielleicht nicht vollständig tadelfrei ist, so kann ich doch diese Angaben durch ähnliche auf andere Weise gewonnene Resultate vollkommen bestätigen.

Bevor ich jedoch Mittheilung von diesen Ergebnissen machen kann, muss ich auf die Beschreibung eines Apparates eingehen, mit Hülfe dessen sie gewonnen wurden und der jedenfalls noch zu verschiedenen anderen Zwecken mit Nutzen verwendet werden kann.

Bekanntlich hat Prof. Bunsen in Band CXLVIII, Heft 3 der Annalen der Chemie und Pharm. in einer Abhandlung "Ueber das Auswaschen der Niederschläge " die Beschreibung eines Filtrirapparates mitgetheilt, womit es durch Anwendung der Wasserluftpumpe gelingt, die Filtration schwer auswaschbarer Substanzen ausserordentlich $\mathrm{zu}$ beschleunigen. Die Leistungen dieses Apparates sind

1) Mémoires de l'acad. royal de méd. de Belgiques. Savants étrangers III. pag. 125,126 u. 177 .

2 Archiv f. path. Anatomie. Bd. 17. S. 421. 422, 
unstreitig ganz vorzügliche um kleine Mengen von Flüssigkeiten zu filtriren, oder grössere Mengen, die nicht allzu viel schleimige Niederschläge enthalten.

Bei physiologischen Arbeiten hat man es nun oft mit relativ grossen Mengen zu thun, deren Filtration, der leicht eintretenden Fäulniss wegen, schnell zu beendigen wünschenswerth ist, oder die wie Blut und Milch nach den bisherigen Methoden überhaupt nicht filtrirbar waren, das heisst kein befriedigendes Filtrat ergaben.

Seit geraumer Zeit beschäftigt mit Untersuchungen über das Serumalbumin, war mir dieser Bunsen'sche Apparat (in seiner Abänderung 1. c. pag. 391 Fig. 7) höchst willkommen und suchte ich denselben sofort für meine besonderen Arbeiten zu verwerthen. Leider stellte sich bei Anwendung desselben heraus, dass das Auswaschen und Filtriren solcher organischen Niederschläge, wie sie durch Ausfällen des Albumin aus dem Serum mittelst Alkohol gewonnen worden waren, zwar einigermassen dadurch beschleunigt werden konnte, dass dasselbe aber dennoch nicht so rasch vor sich gieng, wie es für dergleichen durch allerlei Einflüsse leicht zersetzbare Substanzen zu wünschen ist. - Der Grund einer langsameren und schwierigeren Filtrirbarkeit solcher Stoffe, als der feinvertheilter organischer Niederschläge, liegt theils darin, dass diese schleimigen Massen beim Aussaugen ihrer Suspensionsflüssigkeit sich allzu fest aneinander lagern und festklebend aneinander haften, wodurch das Passiren des Waschungsmittels sehr erschwert wird, theils aber auch, und zwar hauptsächlich, besteht darin, dass die kleine Platinspitze einen zu geringen Angriffspunkt bietet, die saugende Oberfläche zu klein ist.

Um diesem Uebelstande abzuhelfen versuchte ich, auf den Vorschlag von Herrn Geh. Rath Prof. Helmholtz, einen porösen Thoncylinder, wie solche bei constanten Ketten benutzt werden, in ein Filter umzuwandeln. Dieses gelang auch vollständig durch Ueberziehen einer Kautschuckkappe über die Cylinderöffnung, wodurch hermetischer Verschluss von dieser Seite hergestellt wurde. Dieses Thonfilter wurde durch einen luftdichten Gummischlauch mit dem durchbohrten Kautschukpfropfen der hochstehenden Flasche a in Verbindung gesetzt. Am Boden beider Flaschen sind bekanntlich Hähne eingefügt, die durch einen langen Gummischlauch miteinander in Verbindung stehen. Ist die obere Flasche a ausgelaufen, so wird 
der Pfropfen abgenommen, a mit der unteren Flasche $b$ vertauscht und in den Hals dieser der Pfropfen eingesetzt.

Es wurde immer zum Verschluss der Oeffnung der Thonzelle eine Kautschukkappe mit zwei Ansatzröhren gewählt. In eine der beiden Kappenröhren wurde eine genau passende kurze Glasröhre eingeschoben, mit welcher der zum Pfropfen der obern Flasche gehende Gummischlauch in Verbindung stand; in die andere kam eine längere Glasröhre, die bis zum Boden der Zelle reichte und an deren äusseres Ende eine kleine Gummiröhre angesetzt war, welche durch einen Quetschhahn comprimirt gehalten wurde. Letzere Vorrichtung hat den Zweck als Heber zu dienen. Es ist vortheilhaft, vor dem Ueberziehen der Kautschukkappe auf die Oeffnung des Cy. linders ein doppelt durchbohrtes Brettchen zu legen, da sonst die Kappe durch den starken Druck von Aussen nach Innen getrieben und dadurch bald unbrauchbar wird. - Die auf solche Weise zum Filtrum umgewandelte Thonzelle wurde in ein cylindrisches Glasgefäss, wenig weiter als die Zelle selhst gesetzt; dieses Gefäss war bestimmt ausserdem noch die zu filtrirende und auszuwaschende Substanz aufzunehmen.

Soll die Filtration vorgenommen werden, so wird die dazu bestimmte Fliussigkeit in den Apparat gebracht, und zwar so, dass die von der Kautschukkappe unbedeckte Oberfläche des Thoncylinders vollständig von der Flüssigkeit bedeckt wird. Es ist dies um so eher möglich, als man ja, je nach der zu filtrirenden Flüssig. keitsmenge, beliebig grosse Zellen und Cylinder auswählen kann. Hierauf wird der Hahn der hochgestellten Flasche (a) geöffnet und damit das Einsaugen eingeleitet. Soll die im Apparat befindliche Substanz ausgewaschen werden, so kann man, um den Flïssigkeitsstand auf constanter Höhe zu halten, das in einem andern Gefäss befindliche Waschmittel durch eine Heberröhre damit in Verbindung setzen. Beabsichtigt man das auf diese Weise erhaltene Filtrat nicht zur Untersuchung zu verwenden, so kann man dasselbe durch die obere Flasche ansaugen lassen. Will man jedoch das Filtrat selbst untersuchen, so nimmt man dasselbe mittelst des in der Thonzelle befindlichen Hebers heraus. - Das vollständige Abfiltriren der Flüssigkeit, in welcher der zu filtrirende Körper suspendirt ist, wird dadurch begünstigt, dass auf dem von Flüssigkeit entblössten 'Theil der Zelle der Niederschlag durch Ansaugen sich so fest anschlägt, dass Luftzutritt von hier nach Innen vollkommen unmöglich wird, 
Die Filtration solcher schleimiger organischer Stoffe geht nun zwar nicht in so kurzer Zeit vor sich, wie dies nach Bunsen für feinvertheilte anorganische Substanzen der Fall ist, immerhin aber rasch genug, dass bei genügend anderweitigen Vorsichtsmassregeln, Zersetzungen, denen dieselben so leicht unterliegen, nicht stattfinden können. Mittelst dieser Abänderung können aber Substanzen filtrirt werden, die ohne dieselbe geradezu unfiltrirbar zu nennen sind. Bei meinen Untersuchungen betrug die Druckhöhe nur 2,5 Meter, nichtsdestoweniger war das Ergebniss sehr befriedigend, und ist nicht $\mathrm{zu}$ zweifeln, dass bei gehöriger Druckerhöhung die Filtration bedeutend beschleunigt und gesteigert werden kann.

Nach dem jedesmaligen Gebrauch wurden die Thonzellen mit concentrirter Salpetersäure behandelt, sodann in verdünnter Kalilauge und hierauf in Wasser eine Zeit lang gekocht, theils zur Entfernung anhaftender Substanzen, theils zur Frtödtung etwa gebildeter Fermente; ausserdem liess ich nachher noch immer eine Zeit lang reines kaltes Wasser hindurchsaugen. Aehnlich wurde auch die Kautschukkappe mit Glasröhren behandelt.

Diesen Apparat habe ich nur zur Filtration von Blutserum und Milch verwandt. Seine Zweckmässigkeit und Leistungsfähigkeit wird genugsam erhellen, wenn ich anführe, dass Blutserum von den Blutkörperchen und ihren Resten abfiltrirt werden kann und die Milchflüssigkeit von den Fettkügelchen, sowie dass die Suspensionsflüssigkeit von $300 \mathrm{Cc}$. der zu filtrirenden Substanz bei gehörigem luftdichtem Verschluss der mit der Flüssigkeit nicht in Berührung kommenden Theile des Apparates in 4-5 Stunden eingesaugt werden konnte. Die Filtrate beider von mir benutzten Emulsionen waren vollkommen wasserklar und zeigte das Serumfiltrat, vor den Spectralapparat gebracht, von Absorptionsstreifen keine Spur mehr. Von dem Milchfiltrate in Nachfolgendem.

Die zu untersuchende Milch wurde immer von der Kuh weg in den Apparat gebracht und dieser in Eis gesetzt, worin er auch während der Dauer der Untersuchung verblieb. - Die Reaction der Milch war selten schwach alkalisch, noch seltener neutral, meistens aber schwach sauer. Letztere Erscheinung zeigte sich immer bei den erstgemolkenen Portionen, während die letzten meistens schwach alkalisch reagirten, so dass diese drei Zustände in der Milch desselben Euters vorkonmen können. Das Filtrat reagirte immer wie die Milch, woraus es gewonnen worden war. 
In diesem Filtrate war immer, wenn auch oft nur in geringer Menge, ein durch die Wärme coagulirbarer Eiweisskörper vorhanden. Durch Alkohol wird derselbe gefällt; verdünnte Essigsäure bewirkt keine Veränderung in der Flüssigkeit; Salpetersäure ruft anfangs Trübung hervor, die aber durch Ueberschuss der Säure unter Gelbfärbung der Flüssigkeit wieder verschwindet; durch Erwärmen wird die gelbe Farbe intensiver. Kaliumgoldchlorid, ein in jüngster Zeit in der Mikrochemie so beliebtes Reagens, bewirkt in höchst verdünnter Lösung in dem mit Essigsäure schwach angesäuerten Filtrat eine starke Trübung und Gerinnselbildung, und beim Erwärmen eine intensive Violettfärbung. Kurz dieser Eiweisskörper zeigt Eigenschaften, die sich in Nichts von denen des Serumalbumins unterscheiden, so dass beide Proteïnsubstanzen möglicherweise identisch sind, oder sich doch jedenfalls nicht sehr von einander unterscheiden.

Der Gesammtgehalt der Milch an Albumin schwankte in den verschiedenen von mir untersuchten Milchportionen zwischen 0,108$1,450 \%$. Wie ersichtlich stimmt dies mit der von Vernois und Becquerel, Marchand, Hoppe und Anderen bei ihren Milchanalysen erhaltenen Albuminmenge auffallend überein, ohne dass die von mir angewandte Methode den Vorwurf erleidet, als habe hei der Isolirung dieses Körpers bereits eine chemische Veränderung der in normaler Milch vorkommenden Eiweissstoffe stattgefunden. Die Albuminmenge der Milch ist nicht nur bei verschiedenen Thieren verschieden, sondern sogar bei demselben Thier äusserst schwankend. Einige Male konnte jch constatiren, dass die Abendmilch mehr Albumin enthielt als die Morgenmilch.

Die geringe und oftmals so schwankende Albuminmenge der Milch ist es vornehmlich, was einen Zweifel über das Vorkommen dieses Körpers als normaler Milchbestandtheil hervorrufen konnte. Durch die obige Filtrationsmethode und die daraus resultirenden Ergebnisse dürfte nun jeglicher Zweifel gehoben sein. Niemals habe ich bei den vielen von mir angestellten Versuchen ein Filtrat erhalten, das kein Albumin enthalten hätte. Aber auch in der nichtfiltrirten Milch lässt sich sein Vorhandensein nachweisen. Erwärmt man nämlich frische Milch ganz langsam bis sie kocht, lässt sie hierauf eine Zeit lang stehen und giesst sie sodann behutsam aus, so bleiben auf dem Boden des Gefässes kleine Coagula zurück, welche Eiweissreaktion zeigen und kaum etwas anderes als Albumin sein können, da es ja eine charakteristische Eigenschaft des Caseïn ist, 
in der Wärme nicht zu gerinnen. Die Richtigkeit dieser Ansicht beweist folgender Controlversuch.

Von ganz frischer Milch, die etwa eine halbe Stunde kalt gestanden hatte, wurden $30 \mathrm{Cc}$. in den Filtrirapparat gebracht und die Filtration eingeleitet. Das Filtrat hiervon war wie immer vollkommen wasserklar, trübte sich beim Erwärmen stark und schied hierauf Gerinnsel ab. - Andere $30 \mathrm{Cc}$. derselben Milch wurden langsam bis zum Kochen erhitzt, hierauf kühl gestellt und sodann gleichzeitig mit der nichtgekochten Portion in einen gleichen Apparat gebracht. Das Filtrat hiervon war ebenfalls vollkommen klar und zeigte dieselbe Reaction wie die Milch selbst. Erhitzen bewirkte keinerlei Veränderung im Filtrate. Zusatz von verdünnter Essigsäure rief eine nur sehr leichte vorübergehende Trübung hervor; dies war bei Zusatz derselben Säure zum Filtrat der nichtgekochten Milch nicht der Fall gewesen. Durch Salpetersäure entstand eine schwache Trübung, die sich bei Zusatz von viel Säure gelblich färbte und beim Erwärmen unter Beibehaltung der gelblichen Färbung sich wieder löste. Hieraus ergiebt sich nun, dass im Filtrat der gekochten Milch kein Albumin vorhanden und dasselbe bereits beim Kochen der Milch coagulirt war, seiner geringen Menge wegen aber in dieser undurchsichtigen Emulsion nicht wahrgenommen werden konnte. Der im Filtrat in nur äusserst geringen Spuren vorhandene und durch Essig- und Salpetersäure nachweisbare Eiweisskörper dürfte wohl freies Natronalbuminat gewesen sein, das sich beim Kochen der Milch gebildet hatte ${ }^{1}$ ). Dies ist um so wahrscheinlicher, als künstliches Albuminat, wie wir weiter unten sehen werden, leicht in das Filtrat übergeht.

\section{Das Caseïn der Milch.}

Ausser dem Lactalbumin kommt in der Milch noch ein anderer Eiweisskörper vor, und zwar in grösserer Menge als jener, das Caseïn. Diese Proteïnsubstanz wurde lange Zeit hindurch als der specifische und einzige Eiweisskörper der Milch angesehen, und es giebt noch Chemiker, die ihm dies zusprechen. Was nun Letzteres anlangt, so haben wir bereits gesehen, dass dies nicht der Fall ist, sondern dass neben ihm noch ein anderer Eiweisskörper, das Albumin, als normaler Bestandtheil in der: Milch vorkommt; erstere Bezeichnung

1) Kübne, Lehrbuch d. physiolog. Chen. S. 177. 
jedoch kann man ihm immerhin noch zukommen lassen, indem im thierischen Organismus bis jetzt noch kein Eiweisskörper aufgefunden wurde, der vollkommen mit ihm identificirt werden könnte.

Als der am leichtest zugängliche Pateïnkörper der Milch war das Caseïn längst Gegenstand vieler Untersuchungen gewesen und ist dasselbe desshalb wohl auch nach allen seinen Eigenschaften so ziemlich bekannt. Die charakteristischsten sind : dass seine Lösungen in der Wärme nicht gerinnen, Gerinnung jedoch eintritt bei Zusatz von wenig Essigsäure; dass Zusatz von mehr Säure Coagulation in der Kälte hervorruft und Ueberschuss derselben wieder lösung der ausgefällten Substanz zur Folge hat; aus dieser sauern Lösung wird es wieder durch Neutralisiren mit einem Alkali angefüllt. Eintragen von Chlorcalcium und schwefelsaurer Magnesia bewirkt ebenfalls einen Niederschlag. Diese und noch andere Eigenschaften hat es mit dem künstlich hergestellten Kalialbuminat dermassen gemein, dass in Folge dessen die Bezeichnungen Caseïn und Kalialbuminat oft als gleichbedeutend gebraucht und beide Proteïnsubstanzen als identisch angesehen wurden.

Gegen die Identität beider Körper war Hoppe ${ }^{1}$ ), indem er darauf hinwies, dass die Milch weder bei neutraler noch bei schwach saurer Reaction einen Niederschlag absetze und sich ein solcher erst bei weiterer Zunahme des Säuregrades bilde, dass in einer Alkalialbuminatlösung jedoch bei vorsichtiger Neutralisation immer ein Niederschlag eintrete, der erst bei reichlichem Zusatz einer Säure sich wieder auflöst. Ausserdem ist auch die specifische Drehung Beider wesentlich verschieden. Diese Ansicht von der Verschiedenheit beider Proteïnsubstanzen erhält durch Folgendes eine wesentliche Bestätigung.

Weiter oben wurde bereits angeführt, dass verdünnte Essigsäure, dem Milchfiltrate zugesetzt keinerlei Veränderung in dem. selben hervorrief, sondern dass der ganze Eiweissgehalt der Flüssigkeit ohne Säurezusatz durch Erwärmen abgeschieden werden konnte. Es lag nun die Vermuthung nahe, dass der Thoncylinder für das Caseïn wie für die Milchfette undurchgängig sei. Diese Annahme bestätigte sich bald, indem bei längerem Auswaschen des Rückstandes schliesslich keine Eiweisskörper mehr in das Filtrat übergiengen, der Rückstand aber aus Caseïn, Milchfetten und wenigen Salzen

1) 1. c. pag. 418 . 
bestand. Auch Hoppe ${ }^{1}$ ) hat bereits erwähnt, dass in allen seinen Versuchen die Quantität des Caseïn im Transsudate nur eben so gross oder noch geringer als das Albumin erschien, so dass das erstere noch grösseren Widerstand als letzteres beim Durchtritt thierischer Membranen zu finden scheint. Anders verhält es sich in dieser Hinsicht mit dem künstlichen Kalialbuminat, denn eine Lösung von diesem in den Apparat gebracht filtrirt eben so rasch als Serum- und Lactalbumin. Es ist nun zwar sehr möglich, dass das Caseïn in der Milch nicht in Lösung enthalten ist, sondern irgendwie eine Verbindung zwischen ihm und den Fetten besteht. Für diese Ansicht spricht auch die ganze Art und Weise der Milchgerinnung, indem bei diesem Vorgang ja Caseïn und Fettkügelchen die leberartigen Coagula bilden, während das gelöste Albumin in dem ausgepressten Serum enthalten ist. Immerhin aber ist die Undurchgängigkeit der Thonzelle für das Caseïn und seine Durchgängigkeit für das künstliche Kalialbuminat ein wesentliches Unterscheidungsmoment beider Proteïnkörper.

Ebenso verschieden ist das Verhalten dieser beiden Körper gegen die Einwirkung von kohlensauren Alkalien.

Das Gerinnen der Caseïnlösungen in der Wärme bei Gegenwart von kohlensaurem Kali oder von Salpeterlösung, die mit etwas Kali versetzt worden war, hatte bereits $L$ ehman ${ }^{2}$ ) beobachtet und sich diese Erscheinung auf eine höchst complicirte Weise zu erklären versucht, da er sich nicht überreden konnte, diesen Körper wfür eine in blosser Hitze coagulirbare Modification des Caseïn anzunehmen (wie solche auch zuweilen in der Milch enthalten zu sein scheinen)«. Ohne diese Beobachtung Leh man n's zu kennen, hatte ich auf Grund der von Setschenow ${ }^{3}$ ) und Pflü ger ${ }^{4}$ ) angestellten Gasanalysen und der daraus erhaltenen Resultate Versuche über die Einwirkung verschiedener Salze auf frische normale Milch angestellt. Die angewandten Salze waren kohlensaures und schwefelsaures Natron und Chlornatrium. Es wurden von diesen Salzen immer 10procentige Lösungen benutzt, nur bei Chlornatrium wurde auch eine 20procentige angewandt, da durch die andere keine Ver-

1) 1. c. pag. 423 .

2) Lehrbuch der physiol. Chemie. 2. Auf. S. 384 .

3) Zeitschrift f. rat. Medicin von Henle u. Pfeuffer. Dritte Reihe. Bd. X. p. 285.

4) Dieses Archiv Bd. II. S. 172. 
änderung erzielt wurde. Die Milch wurde, bevor Versuche damit angestellt wurden, immer genau neutralisirt; einige Mal war dies gar nicht nöthig. $\mathrm{Zu}$ je $2 \mathrm{Cc}$. der Milch wurden immer um 0,5 Cc. grössere Portionen der Salzlösungen gebracht. Die bei verschiedenen Versuchsreihen enthaltenen Resultate blieben sich immer gleich.

\begin{tabular}{|c|c|c|c|c|c|c|c|}
\hline $\begin{array}{l}10 \text { pCt. } \\
\text { koblhon- } \\
\text { saureg } \\
\text { Natron }\end{array}$ & & $\begin{array}{l}\text { 10 pCt, } \\
\text { schwe- } \\
\text { felsuaur. } \\
\text { Natron }\end{array}$ & & $\begin{array}{l}10 \text { pdt. } \\
\text { Chlor- } \\
\text { natr. }\end{array}$ & & $\begin{array}{l}20 \text { pCt, } \\
\text { Chlor- } \\
\text { natr. }\end{array}$ & \\
\hline Cc. & & Cc. & & Cc. & & Cc. & \\
\hline \multirow[t]{2}{*}{0,5} & Gelbfärbung & 0,5 & - & 0,5 & -- & 0,5 & - \\
\hline & b. Erwärmen & 1,0 & - & 1,0 & - & 1,0 & - \\
\hline 1,0 & - & 1,5 & Leichte Trüb. & 1,5 & $\ldots$ & 1,5 & Trüb. b. Erw \\
\hline \multirow{2}{*}{1,5} & Trübung beim & & b. Erwärmen & 2,0 & - & 2,0 & Gerinn. b.Er \\
\hline & Erwärmen & 2,0 & - & 2,5 & - & 2,5 & - \\
\hline \multirow[t]{2}{*}{2,0} & Gerinnung $\mathbf{b}$. & 2,5 & - & 3,0 & - & 3,0 & - \\
\hline & Erwärmen & 3,0 & Starke Trüb. & 3,5 & - & 3,5 & - \\
\hline 2,5 & - & & b. Erwärmen & 4,0 & - & 4,0 & - \\
\hline \multirow[t]{2}{*}{3,0} & Trübung & 3.5 & - & 4,5 & - & 4,5 & - \\
\hline & in der Kälte & 4,0 & - & 5,0 & - & 5,0 & - \\
\hline 3,5 & - & 4,5 & - & 5,5 & $\bar{\pi}$ & 5,5 & $\bar{\pi}$ \\
\hline \multirow[t]{2}{*}{4,0} & $\begin{array}{l}\text { Gerinnung } \\
\text { in der Kälte }\end{array}$ & $\begin{array}{l}5,0 \\
5,5\end{array}$ & - & 6,0 & $\begin{array}{l}\text { Keine Verän- } \\
\text { derung b. Erw. }\end{array}$ & 6,01 & $\begin{array}{l}\text { Keine Veränd. } \\
\text { in der Kälte }\end{array}$ \\
\hline & - & 6,0 & $\begin{array}{l}\text { Deutliche Ge- } \\
\text { rinu. b. Erw. }\end{array}$ & & - & & - \\
\hline
\end{tabular}

Hieraus geht nun deutlich hervor, dass ganz besonders Zusatz von kohlensaurem Natron zur Milch eine sehr wesentliche Modification derselben bedingt, indem seine Anwesenheit Gerinnung der vorher in der Wärme nicht gerinnenden Milch veranlasst. - Nicht eben dieselbe Erscheinung tritt aber bei Zusatz von kohlensaurem Natron zu einer Lösung von Kalialbuminat auf, denn diese erleidet weder in der Kälte noch in der Wärme irgendwelche Veränderung.

Diese beiden sehr wesentlichen Unterschiede des Caseïns und Kalialbuminats lassen sehr wohl die Ansicht zu, dass beide Körper nicht identisch sind und dass Caseïn keineswegs gleich Kalialbuminat zu setzen ist.

Ueber das Entstehen dieses eigenthümlichen Eiweisskörpers der Milch sind schon allerlei Meinungen geltend gemacht worden, bald hielt man ihn für ein blosses Transsudat aus dem Blute, bald wieder für ein Produkt der Milchdrüse, konnte aber weder für das Eine noch für das Andere Beweise beibringen. So viel mir bekannt ist, war Kemmerich ${ }^{1}$ ) der Erste, welcher die nächstliegende Vermuthung aufstellte, dass dieser Körper aus dem Milchalbumin ent-

1) E. Kemmerich, Beiträge ;zur Kenntniss der physiol. Chemie der Milch. Centralblatt f. d. medicin. Wissenschaften. 1867. Nr. 27 u. dieses Archiv Bd. II. S. 401. 
stehe und der diese Ansicht auch durch Beweise bekräftigte, indem er den Caseïn- und Albumingehalt frischer Milch bestimmte und die Vermehrung des ersteren bei Verminderung des letzteren nach einigen Stunden Digestion derselben Milch nachwies.

Im Laufe dieser Untersuchungen beschäftigte ich mich auch mit dieser Frage und kam zu demselben Resultate wie Kemmerich, so dass ich dessen Angaben vollkommen bestätigen kann. Bemerken muss ich jedoch, dass ich nicht wie er mit frischer Milch arbeitete, sondern mir, nach der von De $\mathrm{n}$ is ${ }^{1}$ ) angegebenen Methode, Magnesiamolken darstellte und diese zu meinen Versuchen benutzte; ausserdem stellte ich noch mit dem nach obiger Weise aus frischer Milch gewonnenen Filtrate Versuche an. Liess ich beide Flüssigkeiten, in welchen verdünnte Essigsäure anfangs keine Veränderung bewirkte, eine Zeit lang im Brütofen bei $38^{\circ} \mathrm{C}$. digeriren, so liess sich die Caseïnbildung in derselben sowohl qualitativ als auch quantitativ nachweisen. Diese Caseïnbildung war immer mit dem Eintritt der Milchsäuregährung in der vorher neutralen Flüssigkeit verbunden. Liess man die Probeportion längere Zeit digeriren, so dass sich eine genügende Menge freier Säure bilden konnte, so fiel das Caseïn von selbst aus, ohne vorherigen Essigsäurezusatz. Niemals gelang es mir, wie ich beabsichtigte, sämmtliches Albumin durch Digestion in Caseïn überzuführen. Möglicherweise würde dies durch zeitweilige Neutralisation gelungen sein, da ja bekanntlich durch Anwesenheit freier Säure das Fortschreiten der Milchsäuregährung sehr gehindert wird ${ }^{2}$ ).

Es ist eine bekannte Thatsache und wurde meines Wissens zuerst von $\mathrm{Hoppe} \mathrm{e}^{3}$ ) darauf hingewiesen, "dass frische normale Milch von Kühen und Ziegen beim Kochen trotz alkalischer Reaction gerinnt«, und glaubt derselbe dies einem wahrscheinlich hohen Albumingehalt der Milch zuschreiben zu dürfen. Leider besitzt man von derartiger Milch keine Analysen und besonders keine gasometrischen. Aus letzteren hätte sich nämlich ergeben müssen, was sehr interessant $\mathrm{zu}$ wissen wäre, ob in solcher Milch chemisch gebundene Kohlensäure, resp. kohlensaure Alkalien vorkommen oder

1) Nouvelles récherches sur le lait. Siehe Henle u. Meissner, Bericht über d. Fortschritte d. Anat. u. Physiol. 1856. S. 274.

2) Archiv f. path. Anat. u. Physiol, Bd. 17. S. 429.

3) 1. c. pag. 419 . 
nicht. Bekanntlich kommen solche, wie aus den bereits erwähnten Untersuchungen von Setschenow und Pflüger hervorgeht, in frischer normaler Milch nicht vor, sondern es konnte von beiden Forschern nur freie Kohlensäure in diesem Secrete nachgewiesen werden.

Wir haben bereits gesehen, dass Zusatz kohlensaurer Alkalien zur Milch beim Erwärmen Gerinnung derselben hervorruft. Die Erklärung dieser Thatsache dürfte wohl schwierig sein, weun man nicht an den Versuchen von Setschenow und Pflüger einen bedeutenden Anhaltspunkt hätte. Im Blute, dem Träger der Nährstoffe des thierischen Organismus, von dem auch zweifelsohne die Milch abstammt, kommen nachgewiesener Massen kohlensaure Alkalien vor und seine Eiweisskörper haben die Fähigkeit durch Thonwände zu filtriren und beim Erwärmen zu coaguliren. Alles dies findet bei dem Caseïn der Milch nicht statt, und es liegt desshalb die Vermuthung sehr nahe, dass diese abweichenden Eigenschaften desselben durch die Abwesenheit kohlensaurer Alkalien in der Milch bedingt ist.

Es tritt nun in Folge dessen eine Frage heran, auf welche Weise die Abwesenheit kohlensaurer Alkalien in der Milch zu erklären sei und mit dieser hängt der Natur der Sache wegen die der Caseïnbildung eng zusammen.

Die Milchsäuregährung findet bekanntermassen schon in der Drüse selbst in Folge eines Fermentes, das von Pasteur nachgewiesen wurde, statt. Durch diese Milchsäurebildung müssen nothwendiger Weise die leicht zersetzbaren kohlensauren Alkalien in milchsaure umgewandelt werden. Dadurch nur kann das gelöste und in der Wärme coagulirbare Lactalbumin in ungelöster und beim Erhitzen nicht gerinnbare Caseïn umgewandelt werden. Für diese Ansicht spricht auch die weiter oben angeführte Thatsache, dass Bildung des Caseïn aus Albumin in der Magnesiamolke und dem Filtrate der frischen Milch immer mit dem Eintreten saurer Reaction dieser Flüssigkeiten innig zusammenhängt. Einen ganz ähnlichen Vorgang konnte ich bei meinen demnächst zu veröffentlichenden Untersuchungen über das Serumalbumin constatiren. Es bildete sich nämlich bei längerem Behandeln dieses Körpers mit Alkohol in Folge von Essigsäurebildung eine Proteïnsubstanz, die in allen ihren Eigenschaften genau mit dem Caseïn übereinstimmte. Ausserdem muss man noch berücksichtigen, dass in der Milch derjenigen Thiere, 
bei welchen kein Caseïn vorkommt, bei alkalischer Reaction auch immer nur Spuren von Milchzucker ${ }^{1}$ ) vorhanden sind, ein für die Milchsäuregährung jedenfalls sehr hinderliches Moment. Dieser Ansicht scheint Kemmerich's Versuch VIII ${ }^{2}$ ) zwar zu widersprechen; es ist jedoch dieser Widerspruch nur ein scheinbarer. Berücksichtigt man nämlich das Faktum, dass nur eine beschränkte Milchsäurebildıng stattfinden kann, und die Wahrscheinlichkeit, dass eine so schwache Säure wie diese, nur in statu nascendi rasch, nachher aber nur nach langer Einwirkung und unter sehr günstigen Bedingungen denselben Erfolg haben kann, wie ihn starke Mineralsäuren nach kuuzer Einwirkung haben (Syntonin), so kann das Resultat von Versuch VIII keineswegs befremden.

Aus Obigem ist nun leicht ersichtlich, dass ich, wie Kemme$\mathrm{rich}^{3}$ ), die Entstehung des Caseïn einer Fermentwirkung zuschreibe, nicht aber einer direkten, sondern einer indirekten.

Einen Versuch muss ich noch erwähnen, den ich nach Hoppe's ${ }^{4}$ ) Vorgang anstellte, der aber ein etwas abweichendes Resultat ergab. Verschiedene Milchportionen schmolz ich vorsichtlich in starkwandige Glasröhren ein, erhitzte sie dann im Oelbade bis auf $140^{\circ} \mathrm{C}$. und liess sie einige Zeit auf dieser Temperaturhöhe. Bei einigen Portionen trat Gerinnung ein, bei andern von derselben Milch hinwieder nicht. Die Ursache dieser Differenz konnte ich nie feststellen, denn es trat auch bei den geronnenen Portionen keine Verfärbung ein, wie dies bei Hoppe der Fall war. Anfangs bildete bei letzteren das Ganze ein Coagulum, das sich aber allmählich zusammenzog und eine geringe Flüssigkeitsmenge auspresste. Die nicht geronnenen Portionen schieden bei ruhigem Liegen viel Rahm ab und coagulirten nach einigen Tagen spontan.

Setzt man den aus saurer Milch erhaltenen Molken concentrirte Kalilauge zu, so entsteht ein bläschenförmiger Niederschlag, der auf einem Filter gesammelt, Eiweissreaction zeigt. Ueber seine Eigenschaften und besonders über seine Fähigkeit künstliche Membranen zu bilden, gedenke ich weitere Untersuchungen anzustellen.

1) Mémoires de l'acad, roy, de méd. de Belg. etc. III. pag. 126 u. 177.

2) Dieses Archiv Bd. II S, 407.

3) Ebendaselbst.

4) 1. c. 418 u. 424 . 
Schliesslich fühle ich mich verptlichtet, Herrn Geh. Rath Prof. Helmholtz, der mir bei diesen und andern Untersuchungen stets mit Rath und That freundlichst zur Seite stand, hier meinen innigsten und aufrichtigsten Dank auszusprechen.

\section{corrigenda.}

S. 301. Anmerkung, statt kleine lies skleinere "

\$302. Zeile 4 von oben, statt Längeseite lies „Beugeseitex.

๖ 302. Tabellenkopf, statt IV. 1. „VI .

"302. Z. 8 von unten, statt IV I. $V I \propto$.

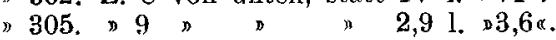

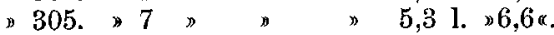

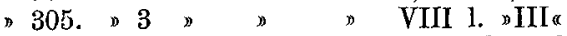

»306. „3 von oben, „3. Phalanx 1 . Handmittea.

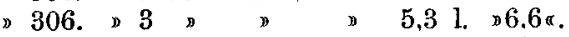

306. \#3 \#)

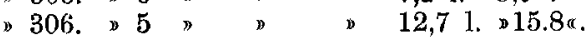

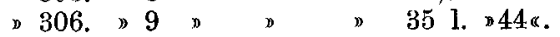

\$26. "18 " " Act 1. "Ast

"426. 13 y, be $1 . \nu$ be .

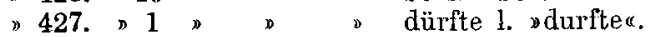

- 429. in der labelle "Procentgehalt 1. "Procentzahla.

, 431. in der Tabelle Z. 2 von unten statt 2,0 1. $\$ 2,5 \alpha$. $\rightarrow 1$ > $10,071.0,72 \pi$ 